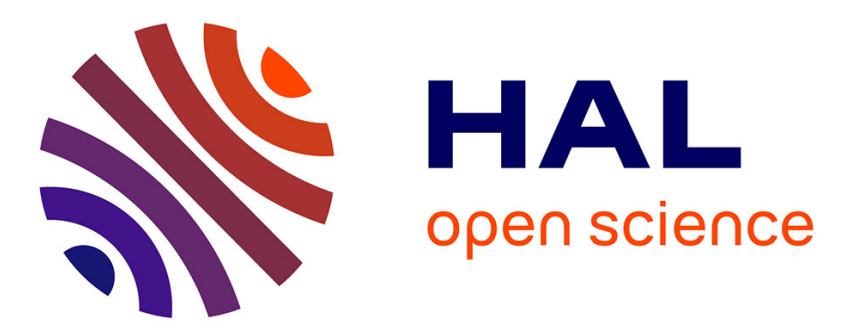

\title{
Logiques politiques et logiques de programme d'action : la création des administrations sociales sous la III $^{\circ}$ République
}

\author{
Didier Renard
}

\section{- To cite this version:}

Didier Renard. Logiques politiques et logiques de programme d'action: la création des administrations sociales sous la III ${ }^{\circ}$ République. Revue française des affaires sociales, 2001, 55 (4, octobre-décembre), pp.33-39. 10.3917/rfas.014.0033 . halshs-00223121

\section{HAL Id: halshs-00223121 https://shs.hal.science/halshs-00223121}

Submitted on 29 Jan 2008

HAL is a multi-disciplinary open access archive for the deposit and dissemination of scientific research documents, whether they are published or not. The documents may come from teaching and research institutions in France or abroad, or from public or private research centers.
L'archive ouverte pluridisciplinaire HAL, est destinée au dépôt et à la diffusion de documents scientifiques de niveau recherche, publiés ou non, émanant des établissements d'enseignement et de recherche français ou étrangers, des laboratoires publics ou privés. 


\section{Logiques politiques et logiques de programme d'action : la création des administrations sociales sous la III $^{\circ}$ République $^{*}$}

Revue française des affaires sociales, volume 55, n 4, octobre-décembre 2001, p. 33-39

\section{Didier Renard}

Une difficulté de l'analyse des politiques publiques est de comprendre comment s'articulent dans la prise de décision les logiques de court terme propres à la vie politique et les logiques de programme d'action qui se développent normalement à terme plus long. L'histoire des créations successives $\mathrm{d}^{\prime}$ administrations centrales en charge des questions sociales sous la III $^{\circ}$ République, outre son intérêt propre, fournit un matériau intéressant pour aborder cette question. L'effet de perspective propre aux terrains historiques favorise $\mathrm{l}^{\prime}$ identification de logiques d'ensemble. L'examen au cas par cas met en évidence au contraire le poids de l'accident et de l'aléa dans la décision. Si l'on raisonne en termes de programmes, en posant que le problème précède la solution, le politique l'administratif, on peut s'attendre en effet à ce que l'institution d'administrations nouvelles signale des politiques nouvelles, à ce que chaque création corresponde à un programme d'action publique identifiable. L'hésitation durable sur les structures, loin d'un modèle d'évolution linéaire, souligne que les liens qu'entretient la politique programmatique avec ces événements organisationnels que sont les créations d'administrations centrales sont moins univoques. La question posée in fine est de déterminer quels éléments s'il en est favorisent, dans le flot de la politique accidentelle et le heurt des projets concurrents, la pérennité des structures administratives ainsi créées. Quatre exemples seront évoqués à l'appui de ce propos : la création de la Direction de l'assistance publique en 1886, puis celles du Ministère du travail en 1906, du Ministère de l'hygiène en 1920 et du Ministère de la santé publique en 1930.

La création de la Direction de l'assistance publique en 1886

En 1886 on réunit au Ministère de l'intérieur, pour former une nouvelle direction d'administration centrale, quatre bureaux jusqu'alors dispersés qui exercent leur tutelle sur des établissements ou des organismes qu'on dirait aujourd'hui à caractère sociale : établissements généraux de bienfaisance, hôpitaux, hospices, bureaux de bienfaisance, services des enfants assistés et des aliénés, sociétés de secours mutuels. Il y a à l'origine de cette création deux projets : l'un de rationalisation administrative, l'autre de développement d'une politique nouvelle.

Le projet de rationalisation administrative résulte du changement de régime politique. Expression de la volonté du Parlement d'exercer maintenant son contrôle sur les administrations centrales, il se traduit notamment par l'obligation pour le gouvernement de fixer par décret les cadres des administrations centrales. L'opération s'accompagne, pour ce qui concerne le Ministère de l'intérieur où elle est effectuée à la fin de 1886, d'une réorganisation des services.

La création d'une Direction de l'assistance publique, qui en est l'élément majeur, relève également de la politique programmatique : elle va dans le sens de la constitution d'une administration de l'hygiène publique, idée qui s'appuie sur un courant d'opinion éclairée et qu'a fermement soutenue René Waldeck Rousseau, Ministre de l'intérieur en 1883-1885. Construire une telle administration suppose au moins de réunir dans un même ensemble les services responsables des hôpitaux, de la médecine gratuite, et de l'hygiène, ce qui impose notamment le transfert du Bureau de l'hygiène du Ministère du commerce vers celui de l'Intérieur. L'opération est formellement réalisée au début de 1889 et solennisée par la modification de la dénomination de la direction, qui devient la Direction de l'assistance et de l'hygiène publiques.

Mais à cette date, un autre programme d'action publique a depuis un peu plus d'un an investi avec succès l'administration créée en 1886, celui de la réactivation du programme de la Révolution

\footnotetext{
* Ce texte est la transcription, légèrement révisée, d'une intervention orale faite au colloque DREES/MIRE sur les Administrations sociales, le 23 mars 2001 à l'Institut International d'Administration Publique. Il s'appuie sur le travail réalisé pour Renard, 2000.
} 
française en matière d'assistance publique. Il s'agit à la fois d'un programme de politique sociale, et d'un programme de laïcisation du social explicitement orienté contre le poids que pèse l'Eglise catholique dans ce secteur d'activité. Ce programme, dont le contenu est lui-même objet de conflits, trouve des soutiens au Parlement, du côté des radicaux, ainsi que dans l'administration, pour une part à la Direction de l'assistance publique, mais surtout à l'Inspection générale du Ministère de l'intérieur, qui compte parmi ses membres des socialistes notoires. Et c'est lui qui, en termes de politiques conduites, donne l'essentiel de son identité à la Direction pour la quinzaine d'années qui suivent, bien que l'opposition du Sénat ne permette pas son entière réalisation.

Dans ce premier cas, la création de l'administration centrale nouvelle résulte de la convergence de projets étrangers l'un à l'autre. Par la suite, il y a bien identification de la Direction à un programme d'action, ce qui favorise sa stabilité organisationnelle et même un certain développement administratif. Mais cette politique n'est pas celle pour la poursuite de laquelle la création de cette administration avait été décidée.

\section{La création du Ministère du Travail en 1906}

Le deuxième exemple est celui de la création, en octobre 1906, du Ministère du travail. Elle répond, ou plutôt renvoie, à une revendication historique importante, récurrente dans la vie sociale et politique française depuis 1848 et portée devant la Chambre des députés à plusieurs reprises depuis le début de la III ${ }^{\circ}$ République. Mais on ne peut dire qu'elle en soit l'aboutissement : elle ne s'exprime pas avec une acuité particulière à ce moment précis. Il existe en revanche, à ce moment précis, une situation politique singulière. Clemenceau, qui comme Ministre de l'intérieur mène depuis quelques mois une politique de répression des mouvements ouvriers, accède à la présidence du Conseil, et il lui est nécessaire de donner des gages à gauche. Le gage est symbolique, mais il est symboliquement fort : c'est la création de ce Ministère mythique, le Ministère du travail. Geste de pure politique donc, qui ne s'accompagne pas d'une réflexion sur les missions du nouveau département et qui ne marque dans l'immédiat aucune inflexion des politiques sociales gouvernementales.

Le nouveau ministère n'est d'ailleurs pas une administration nouvelle. Il est constitué par la juxtaposition de directions d'administrations centrales appartenant au Ministère de l'intérieur et au Ministère du commerce, réunies sans modification de leurs attributions respectives. Coup politique mis à part, le projet qui préside à cette réunion n'est pas évident. Sa logique est celle d'un rassemblement autour de la Direction du travail des administrations d'assistance et de prévoyance, dans la continuité des conceptions sociales qui étaient celles des radiaux dans les décennies 1880-1890. Entre-temps pourtant, le socialisme réformiste d'Alexandre Millerand, ministre du Commerce de 1899 à 1902, avait produit une profonde réforme de l'administration centrale pour en faire, sans pouvoir lui en donner le nom cependant, une administration du travail et de l'assurance sociale. Le Ministère de 1906 constitue par comparaison un recul vers des conceptions plus traditionnelles et l'édifice construit par Millerand est partiellement démembré pour donner naissance au nouveau département qui n'en accueille qu'une partie.

C'est donc un Ministère sans projet unificateur et sans grande puissance financière ni organisationnelle qui est créé en 1906. Son existence est fragile, garantie avant tout par l'impossibilité politique d'un retour en arrière. Elle est confortée par la suite par le développement, avec et après le vote de la loi sur les Retraites ouvrières et paysannes en 1910, d'administrations de la protection sociale. La mise en oeuvre de le loi de 1910 s'accompagne de la création d'une direction d'administration centrale supplémentaire. Entre les deux guerres, celle des assurances sociales contribue fortement à la croissance organisationnelle du ministère.

Dans ce cas, l'écart est donc important entre les déterminants politiques à court terme de la création du Ministère et les déterminants structurels de sa stabilisation puis de sa croissance administrative. Celle-ci une fois entamée, sur des bases qui n'avaient été ni voulues ni prévues au moment de la décision initiale, sa logique de développement s'avère suffisamment puissante et autonome, suffisamment souple également, pour résister avec succès à l'aléa politique. L'existence du Ministère du travail n'est plus remise en question. 
Le troisième exemple est d'une autre nature. Il s'agit, au début de l'année 1920, de la création d'un ministère de l'hygiène, dont la dénomination complète est Ministère de l'hygiène, de l'assistance et la prévoyance sociale, qui emprunte au Ministère du travail la Direction de la mutualité, et au Ministère de l'intérieur les services de l'assistance et de l'hygiène publiques. On est cette fois plus proche de la séquence idéale de la décision, où l'identification d'un problème précède la définition d'un programme, puisqu' on trouve à l'origine de cette création l'irruption sur la scène politique de l'émotion publique que provoquent les épidémies de grippe espagnole, puis de typhus et de peste. L'appareil administratif de protection sanitaire, qui n'arrive pas à y répondre, est mis en cause, et l'on observe la formation d'un consensus à la française autour de l'idée que seule une organisation hiérarchique centralisée dans les mains de l'État pourrait lutter efficacement contre ces fléaux. Il faut donc établir un Ministère de l'hygiène.

Peut-on dire pour autant que c'est le développement des épidémies qui provoque la création du Ministère ? En termes de causalité directe et conjoncturelle, certainement oui : s'il n'y avait pas eu épidémie, il n'y aurait très probablement pas eu ministère. Le projet est cependant largement antérieur à l'événement qui provoque sa réalisation. La configuration administrative du nouveau département est celle qui avait été initialement prévue, puis abandonnée, pour la création de la Direction de l'assistance publique en 1886. Et le projet d'un Ministère de l'hygiène publique, parallèle et concurrent de celui d'un ministère du travail depuis une quarantaine d'années, trouve, favorisé par les circonstances de la guerre, une vitalité renouvelée à partir de 1915. Des propositions de loi sont déposées en ce sens, tandis qu'est évoquée l'idée d'un Ministère des sports qui inclurait l'administration de l'hygiène. L'épidémie survient donc dans un climat déjà favorable à la réforme, comme un facteur déclenchant.

Si les circonstances permettent de faire un geste politique et symbolique qu'on peut estimer de même nature que celui de 1906, elles ne permettent pas en revanche de faire plier les résistances qui ont jusqu'alors empêché la réalisation du projet. La demi-douzaine de départements ministériels qui possèdent en matière d'hygiène des prérogatives ou même des services refusent d'en être dessaisis. Le programme administratif de regroupement des services n'est donc pas mené à bien, le développement d'une politique de santé publique dont il devait être l'appui non plus. Un an après la création du ministère, les critiques parlementaires sont virulentes et le diagnostic sévère : "Le Ministère de l'hygiène a donc désormais une existence légale et théorique, mais jusqu'ici son œuvre est nulle. »

Pour assurer la pérennité d'un département dès sa naissance menacé de disparition, le ministre en fait le vecteur d'un autre programme d'action, qui ne manque pas lui non plus de soutiens au Parlement à ce moment, celui d'une politique de la natalité. L'installation d'un Conseil supérieur de la natalité va en ce sens. Mais c'est alors sa propre administration centrale qui fait défaut au ministre. S'opposant à un Conseil supérieur qui ne comprend aucun fonctionnaire et qui fait montre d'une connaissance parfois approximative des textes en vigueur, elle freine sur ce point l'élan politique du gouvernement.

Le nouveau département ministériel se trouve donc pris en tenaille entre une incapacité à réunir les services administratifs dont le concours est nécessaire à la politique de l'hygiène pour laquelle il a été créé, et la résistance de ses propres fonctionnaires au projet alternatif d'une politique de la natalité. Il reste ainsi particulièrement fragile et ne résiste pas à la vague d'économies budgétaires de 1924, date à laquelle il est supprimé.

\section{La création du Ministère de la santé publique en 1930}

Le dernier exemple est celui de la recréation, en 1930, du Ministère de l'hygiène sous l'appellation plus moderne de Ministère de la Santé Publique. Programmatiquement parlant le projet n'est, au moment où il est créé, pas abouti et pour tout dire fort incertain, même s'il correspond aux vues et à la volonté affichée du Président du conseil, favorable à l'idée d'un ministère de l'hygiène. Sur le plan des capacités administratives, il n'est guère ambitieux, puisqu'il s'agit purement et simplement d'ériger au rang de ministère la toujours jeune Direction de l'assistance et de l'hygiène publiques, et elle seule. 
Mais le projet rencontre le souhait des administrateurs de cette direction, qui ne se sont jamais adaptés aux us ni aux coutumes du Ministère du travail auquel ils ont été rattachés en 1924.

Ces soutiens internes ne se révèlent pas suffisants pour que se développent une administration ni une politique véritablement actives. L'assistance publique, comme mode de protection sociale, est en effet maintenant reléguée à l'arrière- plan par les assurances sociales dont la mise en œuvre par le Ministère du travail est en cours. Sur le versant de l'hygiène ces mêmes assurances sociales, en consacrant la conception libérale de l'exercice de la médecine, portent un coup très rude au projet de développement d'une médecine de santé publique. Au-delà même, le choix politique de l'entier ralliement à l'exercice libéral, cohérent avec l'état de l'opinion parlementaire et qui permet le vote de la loi mais qui s'effectue contre les réticences de l'administration centrale, obère la capacité de celle-ci à conduire une politique de santé dont elle soit le véritable maître d'oeuvre.

Privée en matière d'hygiène publique comme en matière de protection sociale de la capacité de conduire de façon autonome des politiques cohérentes, l'administration de la santé publique reste donc une administration faible dont le maintien de l'autonomie pendant quelques années doit plus à l'inertie politique et à l'opposition de ses personnels d'encadrement à tout rapprochement avec le Ministère du travail qu'à l'existence d'un programme propre. Réduite à un rôle secondaire, elle gère pour les services et établissements dont elle a la charge et par lesquels elle existe, en particulier les hôpitaux, les conséquences des politiques sur lesquelles elle est à peine consultée, du Ministère du travail.

\section{Aléa des créations et pérennité des structures}

On peut en conclusion avancer quelques remarques, qui relèvent du simple bon sens autant que de l'analyse, sur les conditions qui favorisent la pérennité de structures administratives dont la création est si fortement marquée par des éléments de circonstance. Certaines sont d'ordre organisationnel. Les segments d'administration, bureaux ou directions, qui gèrent ou contrôlent des dispositifs instaurés par voie législative ont par construction une existence aussi pérenne que ceux-ci, et un poids directement fonction de leur importance. La mise en oeuvre d'une législation nouvelle permet donc habituellement une certaine expansion des services concernés en même temps que de réajustement des influences en leur sein, bien que l'évolution des moyens, notamment en personnel, ne suive pas en général, pour la période qui nous concerne, l'élargissement des attributions. Ainsi la loi de 1910 sur les retraites ouvrières et paysannes, puis celle de 1928-1930 instituant les assurances sociales, contribuent-elles fortement à l'affirmation administrative du Ministère du travail récemment créé. Les bureaux qui exercent leur surveillance sur des institutions durables ne peuvent disparaître, et la création de structures nouvelles, directions ou ministères, s'analyse à leur égard comme une simple réorganisation qui pose à nouveau la question de leur rattachement, comme dans le cas des services de la mutualité.

Un autre facteur organisationnel déterminant pour la pérennité des administrations centrales est, pour des raisons diverses, l'étendue des services extérieurs qui leur sont attachés. Une direction est d'autant plus puissante et stable que ses services extérieurs sont importants. L'exemple type est sur ce point celui de la Direction du travail organisée dès l'origine autour de l'Inspection du travail. Aussi les directeurs d'administration centrale avisés visent-ils à la création et à l'expansion de ces services, comme on l'observe à la Direction de l'assistance publique où les premières tentatives en ce sens remontent à 1887, et leur faible essor signe-t-il, comme au Ministère de l'hygiène, une faiblesse administrative durable.

Comme on le voit, les logiques de développement des services sont pour une part autonomes et ne dépendent pas entièrement de la fortune des programmes qu'elles sont supposées mettre en oeuvre. Ces programmes existent pourtant et, pour la plupart d'entre eux, font preuve d'une grande longévité. Constamment concurrents, rarement abandonnés, ils perdurent souvent après que les raisons pour lesquelles ils ont été imaginés ont disparu et sont mis en sommeil pendant que d'autres se constituent sur des bases nouvelles. C'est à ce point qu'intervient l'aléa politique, qui modifie entre eux la hiérarchie des priorités et opère à certains moments et pour une variété de raisons de véritables choix, 
cependant réversibles. Cet aléa est puissant. Aussi, parmi les innovations institutionnelles ou organisationnelles qui sont en effet réalisées, celles qui semblent avoir le plus de chance de durer, celles qu' on voit avoir une histoire, sont les plus souples, les plus potentiellement polysémiques, celles qui sont susceptibles de prendre sens au sein d'une variété de projets et de situations, d'y être réinterprétées et d'y servir d'autres objectifs que ceux qui leur avaient été assignés lors de leur création.

\section{Pour en savoir plus}

Friot (Bernard), 1998, Puissances du salariat. Emploi et protection sociale à la française, Paris, La Dispute Hatzfeld (Henri), 1971, Du paupérisme à la sécurité sociale 1850-1940, Paris, Armand Colin [réédition Presses Universitaires de Nancy, 1989]

Renard (Didier), 2000, Initiative des politiques et contrôle des dispositifs décentralisés. La protection sociale et l'Etat sous la III République 1885-1935, rapport de recherche GAPP pour la MIRE 